\title{
Measurement of Semiconductor Surface Potential Using the Scanning Electron Microscope
}

\author{
Jennifer T. Heath \\ Linfield College \\ Chun-Sheng Jiang \\ National Renewable Energy Laboratory \\ Mowafak M. Al-Jassim \\ National Renewable Energy Laboratory
}

Follow this and additional works at: https://digitalcommons.linfield.edu/physfac_pubs

Part of the Physics Commons, and the Semiconductor and Optical Materials Commons

\section{DigitalCommons@Linfield Citation}

Heath, Jennifer T.; Jiang, Chun-Sheng; and Al-Jassim, Mowafak M., "Measurement of Semiconductor Surface Potential Using the Scanning Electron Microscope" (2012). Faculty Publications. Published Version. Submission 3.

https://digitalcommons.linfield.edu/physfac_pubs/3

This Published Version is protected by copyright and/or related rights. It is brought to you for free via open access, courtesy of DigitalCommons@Linfield, with permission from the rights-holder(s). Your use of this Published Version must comply with the Terms of Use for material posted in DigitalCommons@Linfield, or with other stated terms (such as a Creative Commons license) indicated in the record and/or on the work itself. For more information, or if you have questions about permitted uses, please contact digitalcommons@linfield.edu. 


\section{AIP Appilied Physics}

\section{Measurement of semiconductor surface potential using the scanning electron microscope}

Jennifer T. Heath, Chun-Sheng Jiang, and Mowafak M. Al-Jassim

Citation: J. Appl. Phys. 111, 046103 (2012); doi: 10.1063/1.3684556

View online: http://dx.doi.org/10.1063/1.3684556

View Table of Contents: http://jap.aip.org/resource/1/JAPIAU/v111/i4

Published by the American Institute of Physics.

\section{Related Articles}

Transducer for mechanical impedance testing over a wide frequency range through active feedback Rev. Sci. Instrum. 83, 025001 (2012)

Performance of a fast digital integrator in on-field magnetic measurements for particle accelerators Rev. Sci. Instrum. 83, 024702 (2012)

Generalized four-point characterization method using capacitive and ohmic contacts Rev. Sci. Instrum. 83, 024703 (2012)

Extracting accurate capacitance voltage curves from impedance spectroscopy Appl. Phys. Lett. 100, 042101 (2012)

Electrical test method using high density plasmas for high-end printed circuit boards Rev. Sci. Instrum. 83, 013503 (2012)

\section{Additional information on J. Appl. Phys.}

Journal Homepage: http://jap.aip.org/

Journal Information: http://jap.aip.org/about/about_the_journal

Top downloads: http://jap.aip.org/features/most_downloaded

Information for Authors: http://jap.aip.org/authors

\section{ADVERTISEMENT}

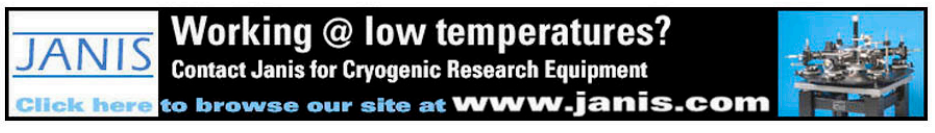




\title{
Measurement of semiconductor surface potential using the scanning electron microscope
}

\author{
Jennifer T. Heath, ${ }^{1, \text { a) }}$ Chun-Sheng Jiang, ${ }^{2}$ and Mowafak M. Al-Jassim ${ }^{2}$ \\ ${ }^{1}$ Linfield College, McMinnville, Oregon 97128, USA \\ ${ }^{2}$ National Renewable Energy Laboratory, Golden, Colorado 80401, USA
}

(Received 10 January 2012; accepted 12 January 2012; published online 21 February 2012)

\begin{abstract}
We calibrate the secondary electron signal from a standard scanning electron microscope to voltage, yielding an image of the surface or near-surface potential. Data on both atomically abrupt heterojunction GaInP/GaAs and diffused homojunction Si solar cell devices clearly show the expected variation in potential with position and applied bias, giving depletion widths and locating metallurgical junctions to an accuracy better than $10 \mathrm{~nm}$. In some images, distortion near the p-n junction is observed, seemingly consistent with the effects of lateral electric fields (patch fields). Reducing the tube bias removes this distortion. This approach results in rapid and straightforward collection of near-surface potential data using a standard scanning electron microscope. (C) 2012 American Institute of Physics. [doi:10.1063/1.3684556]
\end{abstract}

Secondary electron (SE) contrast in the scanning electron microscope (SEM) is known to originate from many different effects, including differing SE yield of materials, crystal orientation, and voltage. ${ }^{1}$ Voltage contrast allows the otherwise identical $\mathrm{p}$ and $\mathrm{n}$ sides of $\mathrm{p}-\mathrm{n}$ junctions to be distinguished. ${ }^{2}$ A SE image across a p-n junction can be quickly and easily collected, and the line section across the junction, illustrated in Fig. 1(a), appears qualitatively similar to the expected variation in surface potential. This has been discussed extensively in the literature, with a goal of imaging relative doping levels in semiconductor materials, resolving small differently doped features, and locating p-n junctions. ${ }^{3}$

Data are often analysed using the relative contrast, $C$,

$$
C=\left(S-S_{\text {ref }}\right) / S_{\text {ref }},
$$

where $S$ is the raw SE signal minus any dark background signal, typically on the p-side of a p-n junction, and $S_{\text {ref }}$ is the reference signal minus the dark background, typically on the $\mathrm{n}$-side of the junction.

Experimental results in the literature show the usefulness of voltage contrast for qualitative studies, but a more detailed understanding is needed to quantitatively verify image features. As a cautionary example, scanning capacitance microscopy images show very clear apparent junction locations, which can be misleading. ${ }^{4}$

Our simple $\mathrm{n}^{+}-\mathrm{p}$ devices allow for measurements both in the vicinity of the junction and in a field-free region away from the junction, as the sample bias and microscope parameters are varied. Based on these results, we have developed an approach for calibrating $C$ to potential (in $\mathrm{V}$ ) for surfaces which are without other sources of SE contrast, such as roughness or strain. These data help clarify the physical mechanism of the contrast and quantify the relationship between contrast and potential.

Although experimental details vary, the consistency of results in the literature, over several decades of effort, is

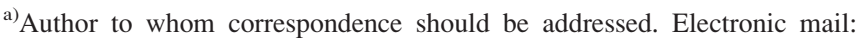
jheath@linfield.edu.
}

quite remarkable. This suggests that voltage contrast depends more on the physical nature of the sample than on the specific experimental equipment used to image it. Recent theoretical work has focused on possible mechanisms for the apparent influence of surface potential on SE collection. ${ }^{5-7}$ These include "patch" electric fields above the sample (PF), which are directed laterally, in the xy plane, ${ }^{8}$ as well as the effective electron affinity $\chi_{\mathrm{E}}$ (EEA). ${ }^{9}$ The EEA is the potential difference between an excited SE just below the sample surface and an equipotential plane, corresponding to a high probability of SE detection, which is determined by the electron optics. Indeed, the PF and EEA effects are intimately interconnected, as one would not arise without the other.

The effect of EEA on SE collection has two parts. First, the kinetic energy of SEs just below the sample surface must be greater than $\chi_{\mathrm{E}}$ such that those electrons will be collected by the detector. Second, diffraction effects occur due to the change in kinetic energy of the SE upon leaving the surface. The electron optics of the microscope have a large, but still limited, collection angle, resulting in a diffraction component of the SE collection efficiency, which depends on the value of $\chi_{\mathrm{E}}{ }^{5}$

In addition, z-direction electric fields arise just below the sample surface, caused by surface band bending (SBB). These can either help propel low energy SEs out of the surface or, alternatively, cause them to be trapped within the sample. ${ }^{6}$

To better understand the relationship between surface potential and SE contrast, we have studied two very different devices, which are both working solar cells.

The heterojunction device ("III-V") is epitaxially grown on a GaAs wafer, shown in Fig. 1(a). At its core are a $1.06 \mu \mathrm{m}$ p-doped GaAs absorber $\left(1.5 \times 10^{17} \mathrm{~cm}^{-3}\right)$ and $90 \mathrm{~nm} \mathrm{n}$ GaInP emitter $\left(1.5 \times 10^{18} \mathrm{~cm}^{-3}\right)$. Layer thicknesses are determined by calibrated deposition rate and times for thin layers, with an accuracy of about $\pm 10 \mathrm{~nm}$, and by SIMS for the thicker GaAs layers. The window/emitter interface is used as a reference to align the data at $x=0 \mathrm{~nm}$, where the positive $x$ direction extends from the emitter into the absorber. 


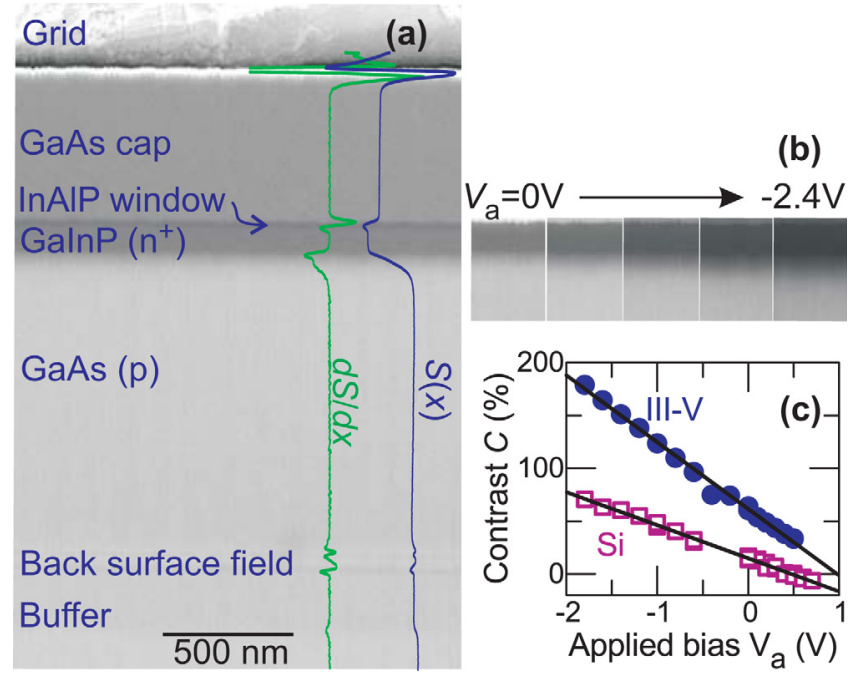

FIG. 1. (Color online) (a) SE image of the III-V sample with line scans of the raw signal $S(x)$ and its spatial derivative overlaid. Peaks in $\mathrm{d} S / \mathrm{d} x$ occur at each metallurgical junction. (b) SE images of the III-V sample with $V_{\text {a }}$ varying from 0 to $-2.4 \mathrm{~V}$ in $-0.6 \mathrm{~V}$ increments. Only the GaInP $\left(\mathrm{n}^{+}\right)$and GaAs (p) layers are shown for clarity. (c) Linear variation of contrast with applied bias in both samples.

In the homojunction device ("Si-U"), the emitter is diffused into a multicrystalline $\mathrm{Si}$ wafer. The SIMS profile of $\mathrm{Si}-\mathrm{U}^{10}$ shows an exponential drop-off in the diffused $\mathrm{P}$ doping, with the metallurgical junction located at $x_{\mathrm{m}}=0.46$ $\pm 0.01 \mu \mathrm{m}$ from the wafer surface and a bulk B doping of $4 \times 10^{16} \mathrm{~cm}^{-3}$. The Si wafers are topped with a $\mathrm{SiN}_{\mathrm{x}}$ antireflective coating, and in the data shown here, the $\mathrm{Si} / \mathrm{SiN}_{\mathrm{x}}$ interface is used as a reference, $x=0$.

The SE data were collected on an FEI Nova 630 SEM at $2 \mathrm{keV}$ beam voltage, $32 \mathrm{pA}$ beam current, and $4 \mathrm{~mm}$ working distance with the immersion lens and in the absence of light. The measured (raw) SE signal varies linearly with beam current. Each sample was freshly cleaved immediately before being placed in the SEM for measurement. Reported data represents the initial exposure of that sample location to the electron beam. This keeps carbon contamination minimal and consistent. For Si-U, smooth areas within crystalline grains were chosen for analysis.

The sample bias was controlled using a custom sample stage and an external voltage supply. In most cases, a bias $-V_{\mathrm{a}}$ was applied to the $\mathrm{n}^{+}$emitter and the $\mathrm{p}$ side of the device was at microscope ground. In other tests, the bias was reversed. We also shorted the $\mathrm{p}$ and $\mathrm{n}$ sides of the sample together and floated them at a bias, $V_{\text {float }}$, relative to the microscope ground, to study the impact of small amounts of bias on the SE optics. For applied bias on the order of a few V, the influence of $V_{\text {float }}$ on $C$ was negligible. For each series of data, other microscope settings were held constant. Consistent with reports in the literature, ${ }^{11} C$ varies linearly with $V_{\mathrm{a}}$, as shown in Figs. 1(b) and 1(c).

The calibration is based on signals $S_{\mathrm{p}}$ and $S_{\mathrm{n}}$ measured outside the depletion region of the $\mathrm{p}$ and $\mathrm{n}^{+}$layers, respectively, as a function of $V_{\mathrm{a}}$. A linear fit to the bulk contrast gives,

$$
C_{0}\left(V_{a}\right)=\left(S_{\mathrm{p}}-S_{\mathrm{n}}\right) / S_{\mathrm{n}}=m V_{a}+b,
$$

as illustrated in Fig. 1(c). The slope, $m$, and intercept, $b$, can then be used to calibrate any raw signal $S$ by replacing $\mathrm{V}_{\mathrm{a}}$ in Eq. (2) with the unknown, calibrated voltage $-S_{\text {cal }}$, where $S_{\text {cal }}$ represents electron potential,

$$
S_{\mathrm{cal}}(V)=-\left(\frac{S-S_{n}}{m S_{n}}-\frac{b}{m}\right) .
$$

Note these are relative voltages such that $S_{\text {p-cal }}(0)=0$. The apparent built-in potential, $V_{\mathrm{bi}-\mathrm{s}}$, is obtained from the intercept at $C_{0}=0: V_{\mathrm{bi}-\mathrm{s}}=-b / m$.

The resulting calibrated line scan is then compared with a simple 1D model of potential variation across the junction created using PC1D software. The model has no free parameters. In both samples, over many experimental repetitions, the quadratic variation of $S_{\text {cal }}$ with distance from the junction corresponds with the model and changes with bias as expected illustrated in Figs. 2 and 3. This indicates that the image is appropriately calibrated.

A spatial derivative of the surface potential, $\mathrm{d} S_{\text {cal }} / \mathrm{d} x$, should be proportional to the $x$ component of electric field, peaking at the metallurgical junction. Then, each metallurgical junction can be clearly identified from the SE image (Figs. 1-3). These results agree closely with the known junction locations. The lateral extent of the electric field within the $\mathrm{n}^{+}$-p junction region corresponds to the depletion width, $W$. The change in $W$ with $V_{\mathrm{a}}$ allows the film doping density to be verified from such images.

For this 1D model to predict the surface potential presumes that $V_{a}$ is applied identically at the surface and in the bulk and that the effective surface doping, influencing the band bending, corresponds to that in the bulk. The close agreement between model and data, especially in Si-U, which has a large density of surface states in the bandgap, suggests that voltage contrast does not strictly yield the surface potential, but rather a near-surface potential.

These data provide interesting input to the discussion of SE contrast. The SBB cannot be a primary mechanism for the contrast in these data. Outside the depletion region, the SBB does not depend on $V_{\mathrm{a}}$; yet, the impact of $V_{\mathrm{a}}$ on $S_{\mathrm{p}}$ is spatially uniform and extends, for $\mathrm{Si}-\mathrm{U}$, throughout the wafer, $0.5 \mathrm{~mm}$ from the junction.

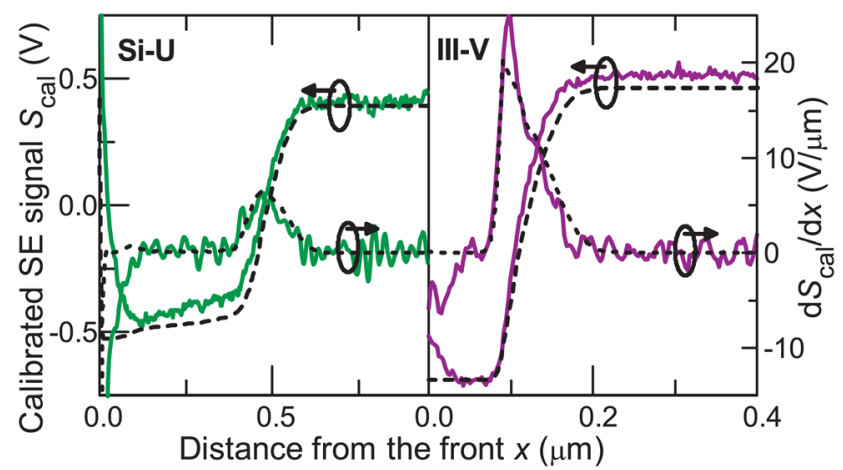

FIG. 2. (Color online) Calibrated SE data (solid line) for Si-U and III-V showing close agreement with the modeled electron potential (dashed line). Curves have been shifted vertically to align. For Si-U, as discussed in the text data collected at $V_{\mathrm{a}}=-0.6 \mathrm{~V}$ is compared to the model for $V_{\mathrm{a}}=0 \mathrm{~V}$. 

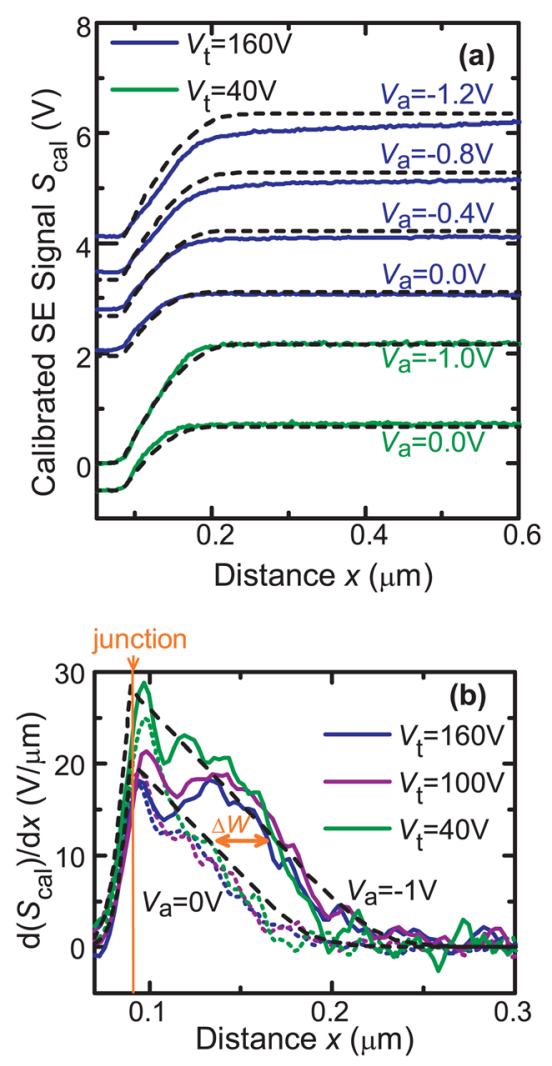

FIG. 3. (Color online) (a) PC1D models (dashed lines) and data (solid lines) for III-V with varying $V_{\mathrm{t}}$ and $V_{\mathrm{a}}$. Traces have been staggered vertically. The distortion is removed by using a lower value of $V_{\mathrm{t}}$. (b) For all values of $V_{\mathrm{t}}$, the metallurgical junction location, depletion width, and its change $(\Delta W)$ with $V_{\text {a }}$ can be easily observed in $\mathrm{d} S / \mathrm{d} x$.

The PF also do not appear to be the primary mechanism for contrast in these data. The spatial extent of the PF predicted by models is more consistent with distortions observed near the junction, such as those illustrated in Fig. 3. ${ }^{7}$ The distortion, observed as a discrepancy between $S_{\text {cal }}$ and the modeled potential, becomes stronger and extends further from the junction with more negative values of $V_{\mathrm{a}}$. This is as expected for PF, which depend on the strength of the lateral electric field. The distortion is reduced by forward bias and in samples with lower $V_{\text {bi-s }}$, such as Si-U. Despite this distortion, the derivative $\mathrm{d} S_{\text {cal }} / \mathrm{d} x$ clearly shows both the metallurgical junction location and the dependence of $W$ on $V_{\mathrm{a}}$, as shown in Fig. 3(b).

This distortion is removed by collecting data at a low tube bias $V_{\mathrm{t}}$ (labeled as "grid bias" in the FEI Nova NanoSEM software), as shown in Fig. 3. The tube bias creates an electric field perpendicular to the sample surface, extracting the SEs; thus, the total SE signal is reduced as tube bias is lowered. At lower $V_{\mathrm{t}}$, changes in $V_{\mathrm{a}}$ would create a larger relative change in $\chi_{\mathrm{E}}$. The reduction of $V_{\mathrm{t}}$ also likely changes the angular and energy distribution of SEs collected. All of these could affect the relative influence of patch fields on the signal.

Another interesting result relates to $V_{\mathrm{bi}-\mathrm{s}}$. We expect SBB to reduce the measured $V_{\text {bi-s }}$ from the bulk $V_{\text {bi }}$ yielded by the model. For the Si-U data shown in Fig. 2, we find $V_{\text {bi- }}$ $\mathrm{s}=0.3 \mathrm{~V}$. This suggests $0.6 \mathrm{~V}$ of $\mathrm{SBB}$, consistent with expected values for $\mathrm{Si}^{7}$ For this reason, the model with $V_{\mathrm{a}}=0 \mathrm{~V}$ is compared to experimental data collected at
$V_{\mathrm{a}}=-0.6 \mathrm{~V}$ in Fig. 2. We were unable to independently measure $V_{\mathrm{bi}-\mathrm{s}}$ in $\mathrm{Si}-\mathrm{U}$ using scanning Kelvin probe microscopy (SKPM), due to the large scale roughness of the sample.

For III-V, we find the difference $V_{\mathrm{bi}}-V_{\mathrm{bi}-\mathrm{s}}$ is less than $0.1 \mathrm{~V}$, so, in Fig. 2, the PC1D model and experimental data are both shown for the same bias, $V_{\mathrm{a}}=0 \mathrm{~V}$. The SBB measured using SKPM is about $0.6 \mathrm{~V}$. A plausible explanation is given by the different degrees of surface Fermi level $\left(E_{F}\right)$ pinning on the two materials. Air absorbates on cleaved Si are expected to generate dense surface states and strongly pin the $E_{F}$, whereas surface defects upon cleaving would weakly pin the $\mathrm{E}_{\mathrm{F}}$ on the GaAs cross-sectional surface. ${ }^{12}$ Unpinning of the surface $E_{F}$ or flattening of SBB would occur on GaAs by the diffusion of excess carriers generated by the high-energy electron beam, whereas the $\mathrm{E}_{\mathrm{F}}$ on $\mathrm{Si}$ would be still pinned at a similar electron exposure. We must in general also consider differing SE yields, as well as the exact depth below the surface at which the contrast is determined, and whether oxides and carbides may play an important role differentiating these two materials' surfaces.

In summary, we have studied the voltage contrast effect in the SEM using two very different devices: a diffused homojunction and an abrupt heterojunction. These simple devices allow for measurements both in the vicinity of the junction and outside the depletion region. By studying the same sample at varying applied bias, the SE signal can be calibrated. Then, the near-surface potential and related information, like depletion width and junction location, can be accurately measured with a standard SEM. This experimental approach also refines our understanding of the underlying physics explaining voltage contrast.

\section{ACKNOWLEDGMENTS}

The authors thank D. J. Friedman for the III-V sample and R. Reedy for SIMS measurements. Thanks also to M. J. Romero, K. J. Kagarice, and R. Passey for useful discussions. This work was supported by the Donors of the American Chemical Society Petroleum Research Fund (J.H.) and the U.S. Department of Energy under Contract No. DE-AC3608GO28308 with the National Renewable Energy Laboratory.

${ }^{1}$ H. Seiler, J. Appl. Phys. 54, R1 (1983).

${ }^{2}$ D. D. Perovic, M. R. Castell, A. Howie, C. Lavoie, T. Tiedje, and J. S. W. Cole, Ultramicroscopy 58, 104 (1995).

${ }^{3}$ P. Kazemian, S. A. M. Mentink, C. Rodenburg, and C. J. Humphreys, J. Appl. Phys. 100, 054901 (2006).

${ }^{4}$ C.-S. Jiang, J. T. Heath, H. R. Moutinho, and M. M. Al-Jassim, J. Appl. Phys. 110, 014514 (2011).

${ }^{5}$ J. Cazaux, Ultramicroscopy 110, 242 (2010).

${ }^{6}$ I. Volotsenko, M. Molotskii, Z. Barkay, J. Marczewski, P. Grabiec, B. Jaroszewicz, G. Meshulam, E. Grunbaum, and Y. Rosenwaks, J. Appl. Phys. 107, 014510 (2010).

${ }^{7}$ A. K. W. Chee, R. F. Broom, C. J. Humphreys, and E. G. T. Bosch, J. Appl. Phys. 109, 013109 (2011).

${ }^{8}$ A. P. Janssen, P. Akhter, C. J. Harland, and J. A. Venables, Surf. Sci. 93, 453 (1980).

${ }^{9}$ C. P. Sealy, M. R. Castell, and P. R. Wilshaw, J. Electron Microsc. 49, 311 (2000).

${ }^{10}$ C.-S. Jiang, H. R. Moutinho, R. Reedy, M. M. Al-Jassim, and A. Blosse, J. Appl. Phys. 104, 104501 (2008).

${ }^{11}$ S. L. Elliott, R. F. Broom, and C. J. Humphreys, J. Appl. Phys. 91, 9116 (2002).

${ }^{12}$ K. J. Choi and J.-L. Lee, Appl. Phys. Lett. 74, 1108 (1999). 